Voix et Images

\title{
" Je suis une femme dans un pays ". Entretien avec Francine Noël
}

\section{Jacques Pelletier et Lori Saint-Martin}

Volume 18, numéro 2 (53), hiver 1993

Francine Noël

URI : https://id.erudit.org/iderudit/201018ar

DOI : https://doi.org/10.7202/201018ar

Aller au sommaire du numéro

Éditeur(s)

Université du Québec à Montréal

ISSN

0318-9201 (imprimé)

1705-933X (numérique)

Découvrir la revue

Citer ce document

Pelletier, J. \& Saint-Martin, L. (1993). « Je suis une femme dans un pays ».

Entretien avec Francine Noël. Voix et Images, 18(2), 224-238.

https://doi.org/10.7202/201018ar d'utilisation que vous pouvez consulter en ligne.

https://apropos.erudit.org/fr/usagers/politique-dutilisation/ 


\section{«Je suis une femme dans un pays». Entretien avec Francine Noël}

Jacques Pelletier et Lori Saint-Martin, Université du Québec à Montréal

\section{I - La venue à l'écriture: Maryse}

V. et I. - Maryse est publié en 1983. Aviez-vous écrit autre chose avant?

F. N. - Non. J'avais bien écrit une thèse de doctorat et un mémoire de maîtrise, mais je n'estime pas que c'était de l'écriture. Je n'avais pas de poèmes dans mes fonds de tiroirs non plus. Mais j'avais collaboré à la revue Jeu pendant un an et demi: je faisais des entrevues, des critiques. J'ai laissé Jeu pour écrire quelque chose de plus personnel. Je trouvais que la critique, c'était une contrainte. Pour arriver à dire ce que je pensais, il fallait inévitablement passer par l'œuvre de quelqu'un d'autre.

\section{V. et I. - Sur quoi portaient les travaux académiques?}

F. N. - Mon mémoire de maîtrise portait sur les contes de Perrault et ma thèse de doctorat sur L'Innommable de Samuel Beckett.

V. et I. - Dans quel type de perspective?

F. N. - La thèse sur Beckett critique la perspective linguistique (Chomsky et compagnie) et le mémoire sur Perrault se situe davantage dans une perspective socio-critique. Il n'y a pas de rapport entre ces deux travaux.

V. et I. - Et y a-t-il un lien avec ce que vous avez fait par la suite? Maryse se moque toujours des contes de fées...

F. N. - Il n'y a pas vraiment de rapport. Je connais assez bien les contes de fées; et l'écriture de Beckett m'a poursuivie pendant des années. C'est un grand écrivain, il est pour moi un modèle, même si je n'écris pas du tout comme lui. Ça m'a pris du temps à m'en détacher: j'ai terminé mon doctorat à vingt-huit ans et j'ai commencé à écrire Maryse deux ans après. Donc il y a eu une espèce de deuil. Pour ce 
qui est de Perrault, c'est un sujet que j'ai choisi davantage en fonction du directeur. J'ai travaillé avec M. Bernard Beugnot, spécialiste du XVII siècle, à l'Université de Montréal, parce qu'il me paraissait être un professeur * civilisé". Je voulais faire mon mémoire avec quelqu'un qui travaille bien et qui soit courtois.

\section{V. et I. - Et où a été écrite la thèse de doctorat?}

F. N. - À Vincennes (Paris VIII), avec Jean-Pierre Richard. J'ai beaucoup détesté faire cette thèse. On m'a suggéré à la soutenance d'aller du côté de la fiction, parce que je n'avais pas une écriture canonique. En effet, j'étais mal à l'aise avec la théorie.

\section{V. et I. - Et vous êtes à l'UQAM depuis quand?}

F. N. - Depuis le début, en 1969, en théâtre.

\section{V. et I. - Et jamais au Département d'études littéraires?}

F. N. - Le théâtre était autrefois avec les Lettres. Au début des années 1970, on nous a demandé de choisir entre rester en lettres ou aller en arts. J'ai choisi d'aller du côté des arts et je n'ai jamais regretté ce choix.

V. et I. - Si Maryse est votre premier texte de fiction, qu'est-ce qui vous a amenée à ce projet?

F. N. - Après la thèse, je n'ai rien produit pendant un an. Puis j'ai commencé un roman que j'ai interrompu parce que je suis devenue enceinte. Après la naissance de mon fils, je suis entrée à la revue Jeu et j'ai découvert là ce que je n'avais pas ressenti lors de mes travaux universitaires: le plaisir de l'écriture. Le plaisir de prendre un texte, de le retravailler, de préciser sa pensée... Au bout d'un an et demi, j'ai annoncé au comité de rédaction que je quittais Jeu pour faire quelque chose toute seule. J'ai alors repris mon vieux manuscrit et j'ai trouvé qu'il n'était pas si mal. C'était l'amorce de Maryse, il y avait déjà là un des trois personnages féminins. Donc, tout en demeurant fascinée par le théâtre, j'ai commencé à écrire des romans.

V. et I. - Maís l'idée de faire ce roman-là, avec ce personnage et la période des années soixante-dix; est-elle venue en même temps que l'idée d'écrire de la fiction, tout simplement?

F. N. - J'ai toujours de longs projets. Je voulais écrire une trilogie. J'imaginais un ensemble romanesque portant sur trois générations: les années quatre-vingt, les années cinquante-soixante et le début du siècle. J'ai comméncé par ce que je connaissais le mieux, les années soixantedix. De cette façon-là, j’avais à vérifier des événements et des dates dans les journaux mais je n'avais pas de vraie recherche historique à 
faire. J'avais l'intention de faire ensuite les deux autres romans, mais en fait j'en ai écrit seulement un, Myriam première, qui se situe dans les années quatre-vingt et qui contient, en abyme, le passé. Dans Maryse, j'ai voulu faire une histoire d'amour, un roman * classique", une histoire qui soit complète, cohérente et convaincante; pour un premier roman, je ne voulais pas jouer avec des téléscopages de temps et de voix. En fait, j'ai repris, sciemment, la figure de Cendrillon.

V. et I. - Il y a donc une certaine naiveté dans ce premier roman. Mais y aurait-il tout de même une certaine influence littéraire derrière: le roman américain contemporain, par exemple?

F. N. - Franchement non. Je connais mal le roman américain contemporain. Je dirais plutôt, sur le plan stylistique, Boris Vian, Raymond Queneau, des gens qui jouent avec la langue, Rabelais... Au Québec, il n'y en a pas beaucoup qui osent le faire, à cause de notre situation de colonisés.

V. et I. - Vous dites que vous avez voulu faire un roman d'amour, mais ce n'est pas du tout comme ça qu'on lit Maryse. On voit plutôt ce texte comme un portrait d'époque et comme l'bistoire de l'êvolution intellectuelle d'une femme. Est-ce que ça renvoie à une expérience personnelle des années soixante-dix? Étiez-vous à ce moment-là dans le syndicalisme, le fëminisme?

F. N. - Dans le féminisme, oui. Pas dans le syndicalisme. Lors des événements d'octobre, j'étais à Paris, je ne les ai pas vécus. Et il y a dans le roman une séquence sur les événements d'Octobre... Je ne sais pas quoi répondre à ça. Ce qui est autobiographique, c'est très mince, par exemple, la mort du père qui laisse un terrain. J'ai écrit mon premier roman à trente-cinq ans; je savais qu'un premier texte est presque toujours autobiographique. Je crois avoir évité cette dimension, ou, du moins, l'avoir suffisamment transposée. Mais je m'illusionne peut-être! De toute façon, cette question ne me tracasse plus maintenant: ma mère est morte, je ne peux plus lui faire de peine, et les interrogations profondes, même mises en veilleuse, constituent la trame de fond et la force des textes qu'on écrit sans tricher. On a beau faire de la fiction, c'est toujours de la vie qu'on parle! Mais ça n'a pas grand chose à voir avec l'anecdote "vécue.

V. et I. - Sans parler de la part d'autobiographie au sens classique, il y a quand même une évocation de ce qui se discutait à ce moment-là, concernant les rapports bommes-femmes, l'engagement politique...

F. N. - J'ai été déléguée syndicale pendant des années, mais je n'ai jamais été membre d'aucun parti, je n'aime pas être enrégimentée. Je 
n'ai jamais eu peur de me définir comme féministe, par contre, parce que je trouve que c'est d'un autre ordre; le féminisme est un des rares mouvements qui aient marqué le vingtième siècle. Ce n'était pas une mode, ça continue et ça a changé la société en profondeur, les hommes autant que les femmes. Mais je n'ai jamais été une féministe militante radicale. Je ne pense pas que ce soit la fonction des écrivains d'être au service d'une cause.

V. et I. - On voit chez vous un mélange, rare au Québec, de féminisme et d'un certain nationalisme.

F. N. - Mais ce n'est pas incompatible: c'est normal d'être une femme et d'être québécoise! Je suis une femme, dans un pays.

V. et I. - Dans un article publié dans Jeu, "Plaidoyer pour mon image ", vous disiez entre autres qu'il n'y a pas beaucoup de bons rôles pour les comédiennes, pas assez de femmes dramaturges... Trouvezvous que la situation a évolué?

F. N. - Je n'ai pas relu cet article, mais c'est le seul que je revendiquerais de tous ceux que j'ai écrits dans Jeu. Ç'a été un texte charnière. J'étais déjà travaillée par le désir d'écrire, de parler en mon nom propre. J'ai beaucoup aimé faire ce numéro sur les femmes, ç'a été une expérience extraordinaire. Je n'écrirais sans doute pas la même chose maintenant, ne serait-ce que parce que je suis de l'autre côté de la clôture et que j'en produis, des images. Et puis, il me semble que la situation a changé, qu'on est plus à l'aise. En général, le féminisme a été absorbé par les créateurs, y compris plusieurs hommes. En ce qui me concerne, quand je me suis mise à écrire - à créer des portraits de femmes - je me suis demandé ce que je faisais. Dans Maryse, par exemple, je donne une image de la mère qui est dure. Mais pour moi c'était inévitable: c'est ce que j'avais le goût d'écrire, c'est ce que j'ai écrit. J'ai eu des interrogations analogues à propos de la représentation des lesbiennes dans Myriam. J'ai beaucoup hésité et j'ai fini par créer deux personnages, une "fine", une "pas fine. La question que je me suis posée est celle-ci: est-ce que c'est utile, socialement, actuellement, de faire un portrait-charge de la lesbienne? Qu'est-ce qui est le mieux: dire ce que j'ai sur le cœur, quitte à ce que ce soit une espèce de cri, peut-être discordant mais authentique, ou ménager la chèvre et le chou, pour ne pas risquer d'être mal interprétée, ou de froisser des susceptibilités? Un texte de fiction n'a pas à être bien pensant ou * politiquement correct . Ce n'est pas l'orthodoxie d'une œuvre qui en assure la force, mais ce qu'elle évoque et provoque, globalement. Bien sûr, il y a une polysémie dans les textes, mais il faut envoyer des messages clairs. Claude 
Gauvreau fait dire à son poète Mycroft qu'il faut poser des gestes tellement clairs que même ceux qui les réprouvent ne puissent en détourner le sens. C'est ce que je vise, mais je ne crois pas y parvenir tout le temps.

V. et I. - Vos deux premiers romans comportatent des registres de langage diffërents: un registre ironique, satirique, accompagnait comme une ombre le registre descriptif. N'était-ce pas une façon de mettre à distance certaines pratiques du milieu intellectuel des années 1970?

F. N. - Mais je percevais vraiment ainsi ce milieu! Je ne pense pas qu'on décide toujours de l'effet qu'on produit. Je ne savais pas que j'étais ironique! D'ailleurs, le plus étonnant, c'est que ma mère, quand elle a lu Maryse, a dit: "Je ne savais pas que ma fille était drôle." Ça signifiait qu'elle ne m'avait jamais écoutée? Peut-être... Chose certaine, nos humeurs nous échappent, et le milieu intellectuel de l'époque me tombait effectivement sur les nerfs. On y pratiquait une forme de terrorisme qui m'agaçait. Je me suis souvenue de Rabelais en écrivant le passage sur l'université dans Maryse. J'ai eu du fun à faire ça, c'est indéniable.

V. et I. - Mais n'avez-vous pas eu peur qu'à un moment donné il y ait un biatus entre les deux registres, et que ça compromette l'unité du roman?

F. N. - Il y a une rupture de ton, je sais, mais ça ne me dérange pas. J'aime les œuvres baroques, et même si je voulais faire un roman straight de Maryse, je me suis permis cet exercice de style. Les lecteurs, ceux qui aiment ce genre de fanfreluches, perçoivent ce passage comme une satire des institutions, en général.

\section{II - Le roman polyphonique: Myriam première}

V. et I. - De vos trois romans, c'est Maryse qui a rejoint le plus large public?

F. N. - Il semble bien. Mais Babel prise deux n'a pas été suffisamment diffusé et Myriam n'a plu qu'à une partie du public. Mais il ne faut pas s'en étonner, c'est construit d'une façon plus sophistiquée que Maryse. C'est une œuvre beaucoup plus savante, ma préférée, bien sûr.

\section{V. et I. - Par comparaison, Maryse est plus spontané?}

F. N. - Non, je l'ai beaucoup travaillé, mais c'est une histoire simple, une chronique. Dans Myriam, je joue avec le temps, je raconte plusieurs histoires en parallèle, le récit est interrompu, c'est complexe. Je 
pense que c'est pour cette raison, en grande partie, que certains lecteurs n'ont pas suivi. C'est qu'ils nous demandent toujours la même chose! Et il y a eu un malentendu: comme je gardais les mêmes personnages, ils ont été déçus. Sauf que moi, ça ne m'intéressait pas de refaire Maryse. Cela dit, il reste que plusieurs préfèrent Myriam. Et ça me fait plaisir. Mais il faut s'attendre à ne pas toujours rejoindre le même genre de monde.

V. et I. - Vous évoquez les différences, mais les deux premiers romans sont quand même très apparentés. Les différences avec Babel sont plus importantes. N'est-ce pas plutôt là que se situe la coupure?

F. N. - Peut-être, je ne sais pas s'il y a coupure.

V. et I. - Pour Myriam, y a-t-il eu certaines influences en ce qui concerne la construction du récit?

F. N. - Les écrivains latino-américains.

V. et I. - Lesquels, précisément?

F. N. - García-Márquez, Isabel Allende, Vargas Llosa, des gens comme ça. Mais je pourrais aussi nommer Sigrid Undset, Jacques Ferron, John Irving...

V. et I. - Pour la construction, Kundera n'a rien à voir?

F. N. - Non, j'ai lu un seul bouquin de Kundera, L'Insoutenable Légèreté de l'être, parce que tout le monde en parlait. C'est très brillant, mais ce n'est pas un auteur dont je me sens proche.

V. et I. - Myriam reprend les mêmes personnages et les mêmes thèmes que Maryse, mais on y sent toutefois les préoccupations d'une nouvelle période, de l'époque postmoderne des années quatre-vingt: la question féministe n'est plus pensée de la même façon. Ce roman a-t-il été conçu comme un projet plus vaste que celui de Maryse?

F. N. - Oui, parce qu'il contient deux romans en un. Il s'agissait de mettre en un mois, en mai 1983, ce qui se passe effectivement en mai 1983, mais aussi le passé de Myriam et de Maryse. C'est beaucoup plus ambitieux comme projet narratif et comme projet de représentation de la société.

V. et I. - Mais c'était volontaire de donner une image à la foìs de l'ensemble des différents milieux des années quatre-vingt et de l'enracinement bistorique, par les personnages des grand-mères?

F. N. - Oui, je voulais faire ça. Moi, je trouve ce roman plus riche que Maryse. Mais je les écris, je ne les lis pas! En fait, quand j'écris, je me demande toujours à qui je vais déplaire... Et chaque fois, il s'agit 
de groupes différents. Donc il doit y avoir une espèce de mouvance du côté des lecteurs.

V. et I. - À cause de la dimension "cbronique "de Maryse, toute une génération a pu s'y reconnaître, alors qu'on peut s'intéresser à Myriam pour d'autres raisons. Ç'a peut-être joué.

F. N. - Oui et non, parce que dans Myriam, il y a les grand-mères, il y a plusieurs milieux, et les lecteurs pouvaient s'y retrouver aussi. Je pense que la date de parution de Maryse a joué: le produit est arrivé sur le marché à un moment où il y avait une sorte de manque, et un peu de nostalgie. Moi je n'étais pas consciente de ça. On m'avait expliqué, à l'Union des écrivains, que huit cents lecteurs, pour un premier roman, c'était très bon, alors je m'attendais à ça, c'est tout.

V. et I. - Une réception plus forte que prévue, c'est agréable!

F. N. - Oui, en principe, c'est agréable.

V. et I. - Ça vous a bloquée pour la suite?

F. N. - Non, ça ne m'a pas du tout bloquée, la preuve, c'est que deux autres romans sont là. Mais c'est dur, de toute façon, de réagir au succès ou à l'insuccès. C'est aussi compliqué dans un cas que dans l'autre. Il y a des gens que le succès rend baveux, d'autres qui se cachent ou qui paniquent. Moi, j'aimerais bien pouvoir me cacher, disparaître dans la brume, même si je sais que c'est puéril.

V. et I. - Est-ce que Myriam a été moins bien accueilli que Maryse par la critique journalistique à l'époque?

F. N. - La presse a été dithyrambique! Sauf une fille, une francophone, qui m'a descendue dans la Gazette. Je dois dire que j'ai été chanceuse, avec la critique.

V. et I. - La figure du poète - Oubedon - change un peu entre les deux romans, non? Dans le premier, il semblait évoquer Miron; dans le second, il fait penser à la poésie New Age, à Claude Beausoleil: c'est juste, cette perception?

F. N. - Adrien Oubedon n'est ni Gaston Miron, ni Claude Beausoleil, ni Michel Beaulieu qui, à l'époque, avait apprécié Maryse, tout en regrettant que je donne cette image des poètes. Pour moi, il ne s'agissait pas de viser la poésie, ou la création en général, ou les intellectuels, et je suis persuadée que les écrivains ont une fonction sociale importante. Gilles Marcotte a écrit un article sur la "poésie Oubedon". Je suis d'accord avec tout ce qu'il dit, mais il y a une chose qu'il ne relève pas et qui est capitale, il me semble: c'est l'existence d'Elvire, la femme du poète, sa muse. La qualité de la production littéraire 
d'Adrien n'est jamais remise en cause, ni son rôle d'homme public. Ce qui est raillé, c'est son comportement privé, son rapport à Elvire et aux enfants. Mais combien d'hommes publics progressistes sont d'indécrassables machos, une fois le micro fermé?! Ce qui n'enlève rien à la qualité de leurs interventions. Mais du point de vue d'une femme, c'est un peu agaçant. Voilà pourquoi j'ai fait d'Adrien Oubedon un personnage complexe: poète à la télé, mais mufle dans la chambre à coucher. Il a du' génie, mais Elvire y est pour quelque chose. J'ai voulu donner à la muse des crédits qu'on lui reconnaît rarement et lui rendre un peu de son espace. Mais c'est toujours d'Adrien qu'on me parle!

v. et I - Pourquoi Maryse est-elle d'origine irlandaise? C'a une signification?

F. N. - Dans la première version, elle était canadienne pure laine. J'ai commencé à écrire Maryse juste après le référendum, j'étais un peu dégonflée. Je me demandais ce que ça allait me donner de faire un personnage de Québécoise, je n'y croyais plus. Et de toute façon, on n'est pas purs, nous, et je ne crois pas à la pureté. J'ai donc fait un personnage métissé car je pense que les Québécois sont "mêlés "... Ma famille compte des Écossais. J'ai choisi les Irlandais parce qu'ils sont déjà présents dans notre littérature. Et sur le plan mythique, l'Irlande est très riche. Je tenais à faire un personnage issu de deux clans; c'est ce qui, très souvent, fait des gens forts, le phénomène d'exogamie.

V. et I. - La récupération du passé, en plus de donner de la dimension et une épaisseur aux personnages, est-elle motivée par des raisons plus larges?

F. N. - Je voulais raconter notre passé, mais sans tomber dans les cretons, la lampe à l'huile, la misère noire... les clichés, quoi! Ce qui m'intéresse, dans les personnages historiques, c'est le mouvement. J'ai voulu tramer et entrelacer plusieurs histoires qui se répondent les unes aux autres comme dans une symphonie.

v. et I. - C'est curieux que le roman s'intitule Myriam, parce que bien que celle-ci soit un personnage important, elle n'a pas la place centrale qu'occupait Maryse dans le premier roman.

F. N. - En fait je me goure toujours dans les titres! Je ne tiens pas du tout à ce titre-là. Je trouve facilement de beaux titres à mes chapitres, mais pas pour l'ensemble.

V. et I. - Pourtant, le titre est important, il programme la lecture...

F. N. - Oui, mais je considère que ça ne fait pas partie du texte. 
V. et I. - C'est peut-être d'ailleurs un problème en ce qui concerne Myriam première, les lecteurs s'attendaient à un pendant de Maryse avec un nouveau personnage féminin. Vous disiez quelque part que vous aviez transfêré sur l'espagnol une partie de vos rapports avec l'anglais. Pourriez-vous préciser?

F. N. - Ma mère parlait anglais par nécessité, pour gagner notre vie, et sachant que la maitrise de deux langues était un atout, elle trouvait les anglophones unilingues bornés et... démunis. C'était pas leur faute, ils n'étaient pas doués pour les langues! Sauf qu'eux pouvaient travailler et être servis sans avoir à traduire leur pensée. Pour eux, pas de coupure entre la maison et la ville. Pour les francophones montréalais, un dédoublement systématique; deux langages: celui de l'intimité et celui qu'on utilise pour se faire comprendre de son propriétaire, de son employeur, à la pharmacie, dans les magasins... Comme si on avait été des immigrants dans notre pays! C'était ça le Montréal des années cinquante: une ville d'abord anglophone. Moi, je n'étais pas sur le marché du travail, mais dans un couvent où la culture francophone nous était transmise sans honte. Pour me rendre à ce couvent, dans l'autobus, je n'entendais que de l'anglais: personne n'essayait jamais de me comprendre, c'était toujours moi qui devais faire toutes les concessions. Alors, j'ai bucké. L'ouverture doit être réciproque, autrement, s'installe un rapport de domination que j'ai perçu d'une façon violente vers dix ans. Ce n'est donc pas une question d'esthétique: en soi, la langue française n'est pas mieux qu'une autre, chaque langue a son charme, sa richesse et ses limites. Non, c'est uniquement un rapport de forces: pour moi, l'anglais se présentait comme la langue du pouvoir, de l'oppression, de la suffisance et de la condescendance. C'était le code utilisé par des gens qui ne voulaient rien savoir... en dehors d'eux-mêmes. Je ne voyais pas et ne vois toujours pas l'intérêt d'entrer en contact avec du monde comme ça. Voilà donc pourquoi je n'ai jamais parlé anglais à Montréal. À Londres, à Paris ou à Old Orchard, oui! Mais pas chez moi. Conséquemment, mon anglais n'est pas des plus riches! J'ai donc fait un transfert - symbolique, comme tous les transferts - vers l'espagnol. C'est un désir d'ouverture, une compensation d'abord émotive; j'ai un fils à moitié espagnol. Mais ce choix correspond aussi à une réalité géopolitique: l'Amérique, en dehors des États-Unis et du Canada, c'est une masse de Latinos. J'aimerais bien être * parfaite bilingue. - français-espagnol - parce que les gens du Sud, quand ils entendent une autre langue que la leur, ils ne restent pas bouche bée comme devant des extra-terrestres: ils essaient de comprendre. Et ça, c'est tellement agréable! Ça vous donne automatiquement l'impression d'exister et le désir de parler la langue de l'autre. 


\section{III - Babel: le choc des cultures}

V. et I. - Que penser alors de Fatima, qui décide, dans Babel, de mener une thérapie en anglais, et de Linda, francopbone de famille péquiste, qui devient anglopbone, en quelque sorte?

F. N. - Ça, c'est un autre aspect de la réalité. On peut très bien être péquiste et imbécile! On peut manquer de cœur dans toutes les langues! Or, quand on est mal prise, comme Linda, il faut être toquée pour refuser l'aide d'une personne sous prétexte qu'elle est d'une autre culture. Linda est un personnage qui veut vivre; elle vivra donc en anglais, puisque son amoureux, d'origine italienne, parle anglais. C'est fort regrettable pour la propagation du français à Montréal et ce n'est pas innocemment que je raconte une telle histoire de cas. L'aphasie de Linda, et l'itinéraire de sa rééducation, ont été pensées comme une métaphore du Québec actuel. Je crois que le roman, dans son ensemble, montre assez bien la dérive dans laquelle nous sommes emportés collectivement. Si jamais on disparaissait, à l'échelle planétaire, "ça ne serait pas si grave. On peut facilement se dire: "Les Chinois font des enfants, alors pourquoi en ferait-on ici? Tout ça, c'est de l'humain.... Peut-être que les espèces disparues ont fait place à d'autres, plus marrantes? Je ne suis pas sentimentale. Cependant, j'ai horreur de tout ce qui évoque la standardisation, l'uniformisation, la réduction. La seule question à se poser, actuellement, à propos de notre "différence", est la suivante: la planète a-t-elle intérêt à un nivellement des cultures particulières au profit de l'imposition d'un magma, sorte de syncrétisme des pratiques des clans dominants? Oublier la saveur du thé servi dans de la porcelaine fine pour ne plus boire que du mauvais café dans un gobelet de styrofoam, ça me semble être d'une tristesse épouvantable! Un appauvrissement. Et puis, il y a le problème du Canada: dans ce "pays", il y a des gens pour qui le simple fait que nous existions, nous du Québec, est insultant... Et puis nous sommes une province d'accueil : c'est bien d'être ouverts à tous - à la planète entière - mais pour ce faire, il faut d'abord être, c'est-à-dire avoir un rapport spécifique au temps, à l'espace, au savoir... Le message global de Babel ressemble à ceci: tenons-nous debout, sachons reconnaître ce que nous sommes et le faire reconnaître. Ensuite on pourra bâtir ce pays, en faire quelque chose. Babel traite donc du phénomène du pluralisme culturel, de la rencontre et du choc de groupes différents. Ce sont des préoccupations actuelles. Que faire avec les nouveaux arrivants? Comment les intégrer sans les assimiler? Je me sens très concernée par ces questions-là, au point que je ne peux pas en parler sans m'enflammer! 
V. et I. - Pourquoi avoir choisi pour ce roman la forme du journal intime?

F. N. - Pour des raisons techniques. Je voulais à nouveau composer une trilogie; une pièce de théâtre, un roman et un film. Le roman devait se passer en 1988; la pièce de théâtre, en 1986; puis le film aurait englobé les deux. J'ai commencé à écrire le roman tout en ébauchant un canevas pour la pièce et un scénario. Pendant trois mois j'ai mené ces trois entreprises de front. Finalement, le roman s'est mieux défendu et s'est imposé à moi comme le livre à faire. Ayant déjà écrit à la troisième personne dans Maryse et Myriam, je voulais cette fois utiliser la première personne. Je n'ai pas réussi à dire "je. autrement que par le biais du journal personnel. Et je voyais très bien Fatima, qui existait déjà dans Cbandeleur, écrire et noter des choses.

V. et I. - S'il y a une coupure stylistique dans votre ceuvre, c'est entre ce roman-là et les deux premiers, qui constituent une sorte d'ensemble. Pourquoi avez-vous renoncé à une représentation polypbonique de la réalité pour adopter le regard très spécifique, très particulier d'un individu sur le monde?

F. N. - J'aime les petits bijoux concis comme Une ardente patience de Skàrmeta. Et comme j'ai tendance à faire long et gros, je me suis posé des balises. Je me suis donné une "commande : écrire un roman de 350 pages en utilisant la première personne et trois personnages seulement. J'ai respecté le contrat. Ce que je voulais faire aussi, c'était montrer des points de vue différents. Il y a de petits passages où ça fonctionne: par exemple, lorsque Louis trouve la pluie merveilleuse alors que Fatima, de son côté, écrit: "Chaque fois que je m'achète des souliers neufs, il pleut! . Je voulais faire ça tout le temps, mais je n'ai pas réussi. J'avais également l'intention de faire une critique des médias et une réflexion sur la langue.

V. et I. - La forme du journal favorise ce glissement vers l'essai.

F. N. - Oui, mais j'ai coupé plusieurs passages théoriques.

V. et I. - Ca ne vous a jamais tenté d'écrire des essais?

F. N. - Bien sûr que ça me tente. Même si je suis loin de mon mémoire et de ma thèse, j'ai une formation qui m'y prédispose. Je pars d'abord, dans mes romans, d'une idée, d'un projet théorique qui me viennent en même temps que des flashes. Tout le travail consiste à mettre en images et en récits cette visée théorique initiale. À l'époque où j'ai écrit Babel, je voulais critiquer les médias mais je me suis rendu compte que ça ne serait pas utile de faire un essai et que le roman me permettrait mieux d'atteindre les mêmes fins. 
V. et I. - Cette dimension est aussi présente dans la représentation qui est proposée des Québécois comme êtres insécures, bonasses, mous, notamment lors de "l'affaire des synagogues "à Outremont. Le point de vue que vous apportez est différent du discours dominant sur cette question.

F. N. - Je suis allée le plus loin possible dans cette direction. Je pensais que les journalistes et les Juifs allaient haïr ce livre. Or j'ai eu un bon accueil des journalistes et pas de réactions de la part des Juifs. J'avais peur d'une intervention du Congrès juif canadien qui a l'habitude de réagir au moindre mot. J'ai beaucoup retravaillé le passage sur les Hassidim. Ce que je tenais à mettre en lumière, c'est l'attitude de repli, de fermeture qu'adoptent les sectes. C'est lourd pour les autres communautés. J'ai longtemps cherché le ton pour l'exprimer et je craignais parfois de déraper.

\section{V. et I. - Mais déraper par rapport à quoi?}

F. N. - Par rapport à la trame narrative. Je craignais d'y perdre en efficacité romanesque avec trop de passages réflexifs. Ils ont du poids dans la mesure où ils sont brefs. Le lecteur, ce qui l'intéresse d'abord, c'est l'histoire de l'amour entre Fatima et Louis.

V. et I. - Donc il y a eu des coupures pour faire ressortir l'bistoire d'amour davantage?

F. N. - Ça démarrait trop lentement. Louis n'entrait pas tout à fait assez vite. J'ai enlevé des références à des événements politiques qui n'avaient pas d'échos dans le reste de l'œuvre. J'ai comprimé surtout le début, et aussi vers les deux tiers, ce qui se passe pendant l'été. Je trouvais que ça languissait, alors j'ai resserré l'action.

V. et I. - Est-ce que la nouvelle version publiée chez Actes Sud sera diffusée ici?

F. N. - Oui c'est un produit Leméac/Actes Sud. J'ai d'abord signé avec Leméac qui a vendu à Actes Sud. Actes Sud a le droit de diffuser en France, en Belgique et en Suisse. Au Québec et partout ailleurs dans le monde, c'est Leméac. Sur la page couverture, le nom Leméac apparaît en premier.

V. et I. - Est-ce qu'il y a eu des ajouts dans cette nouvelle version, ou surtout des coupures?

F. N. - J'ai réécrit quelques passages et j'ai tassé le texte. En tout, j'ai ôté dix pages. Je suis contente de cette version.

V. et I. - Il y a des allusions, dans Babel, et dans une moindre mesure dans Myriam, au rapport à la France. Comment vous situez- 
vous par rapport au débat des dernières années qu'on pourrait résumer par l'affrontement entre Victor-Lévy Beaulieu et Jean Larose?

F. N. - Je n'ai pas suivi ce duel, et les saillies de Lévy Beaulieu me laissent froide. Jean Larose, c'est autre chose, j'aime bien ce qu'il produit même si je suis rarement d'accord avec lui; c'est un polémiste: forcément, il charrie parfois, mais c'est bien, ça nous tient éveillés. Pour ce qui est du rapport à la France, ma position a toujours été la même: je suis d'abord Québécoise. Quand j'ai envoyé Maryse à des éditeurs, c'étaient des Québécois. Il ne m'est pas passé par la tête de l'envoyer en France. J'estime qu'on fait du travail valable ici, et qu'on n'est plus dans les années cinquante. Ce qui ne veut pas dire que je ne désire pas être lue par toute la francophonie. Je trouve déplorable d'aller à Paris et de ne pas voir mes livres dans les kiosques, de ne pas trouver de livres québécois. C'est le problème de tous les francophones vis-à-vis la France. C'est la France qui pose problème, pas nous; elle se comporte comme une puissance colonisatrice. Pour moi, la solution, c'est la coédition, qui ne prive pas les éditeurs québécois de leur droit d'initiative et qui permet une diffusion en Europe.

V. et I. - Vous êtes un peu à mi-chemin entre la survalorisation du Québec cbez Beaulieu et sa dépréciation chez Larose.

F. N. - J'aimerais mieux être définie par rapport à moi-même! Une chose est sûre: jamais je ne déprécierai le Québec, qui m'est toujours apparu comme une heureuse évidence. Je suis Québécoise comme d'autres sont Chinoises ou Parisiennes. Ça n'est pas plus significatif que ça, mais ça l'est autant. Et j'écris depuis Montréal. Si certains Français ne me comprennent pas, c'est leur problème. Dans Babel, Nous avons tous découvert l'Amérique, on a mis un petit lexique à la fin, un glossaire à l'usage des lecteurs européens, ce qui n'est pas très sérieux à mon avis. Et je n'aurais pas accepté de changer mon texte uniquement pour être comprise par des Français. Certaines concessions sont inutiles.

V. et I. - Maryse aurait posé plus de problèmes aú point de vue de la langue.

F. N. - Je ne sais pas... Comme Fatima est une orthophoniste, je lui ai donné une langue plutôt neutre.

V. et I. - $\dot{A}$ travers Amélia, qu'on peut voir comme une passeuse de frontières, Babel soulève la question de la traduction, sujet dont on a peu parlé récemment dans la littérature québécoise.

F. N. - C'est juste. Dans Maryse, il y avait déjà des Espagnols: Maryse se posait des questions sur la femme de Manolo, elle se 
rendait compte que celle-ci ne parlait' pas français. Dans Myriam, Maryse s'en va vers le sud, au Nicaragua. Dans Chandeleur, il y a une petite fille et trois gardiennes, qui sont des archétypes. L'une d'elles, Almira, symbolise la mutante, la "déportée». Je lui fais dire: "Laissezmoi débarquer ici comme vos arrière-grand-mères y sont débarquées [...] il y a à peine trois siècles. " Mis à part les Amérindiens, on est toute une gang de déportés ici: on arrive tous d'ailleurs, que ça fasse cinq jours ou trois siècles. Il faut se le rappeler. Le phénomène d'exil, même si je ne l'ai pas vécu personnellement, m'a toujours émue, ça représente pour moi la déchirure. Et l'exil, ce n'est pas nécessairement l'éviction d'un pays. Il suffit de changer de région ou d'arriver en ville pour se sentir déplacé; nos parents ne sont pas tous des Montréalais de souche, ils sortaient de leur campagne. Il y a des petits pays à l'intérieur d'un pays. C'est un thème qui me fascine et je le reprends dans mon prochain roman. L'action se passera en France et en Espagne, ce sera la dérive du vieux continent vers le nouveau et plusieurs histoires vont s'entrecroiser.

V. et I. - Vous parlez de l'exil, mais vous n'avez pas parlé de traduction. C'est pourtant ce qui frappe dans Babel, où il est question de toutes ces écrivaines inconnues, qui sont le pendant des Sud-américains bommes que tout le monde connait.

F. N. - En fait le personnage qui m'habite depuis des années est celui de la Malinche, dont je parle un peu. Mais chaque fois que j'essaie de la représenter, je n'y parviens pas, tout simplement parce qu'elle n'appartient pas à notre culture, mais à la culture mexicaine. Quand on écrit un texte théorique, on peut généraliser, comparer, faire une digression pour expliquer un détail. Mais quand on fait de la fiction, tout le sens doit être donné par l'œuvre. J'ai connu la Malinche par le biais du texte de Todorov sur la question de l'Autre. La Malinche est la "patronne" des traductrices; en ce sens, elle a aidé Cortés à conquérir Mexico en agissant comme interprète: la traduction, c'est la communication, mais aussi le contrôle. Quand j'écris, j'aimerais pouvoir utiliser plusieurs langues à la fois. Ce qui me fascine, c'est la possibilité de saisir les différents codes, de comprendre les autres. On se définit par rapport à l'Autre.

V. et I. - Une question sur Montréal: que représente cette ville pour vous, comme espace imaginaire?

F. N. - Montréal, c'est tout simplement le lieu où je vis. Si je vivais ailleurs, je parlerais de cet endroit-là. J'aime Montréal, et je ne suis pas gênée de le dire. Comme tout le monde, j'ai des blocages et des angoisses, mais aussi quelques rares certitudes: j'ai toujours été contente 
d'être une femme, d'être une Québécoise et d'être une Montréalaise. Je sais qu'ici, il y a des choses laides; je peux me permettre de les critiquer comme je peux me permettre de les aimer. Dans mon prochain roman, il y aura plusieurs endroits: l'Andalousie, l'Occitanie, l'Amérique du Sud. Des séquences seront situées à Grenade, en 1492, et il restera de la place pour Montréal, rue La Gauchetière. Je suis consciente du fait que je parle rarement de la campagne. Pourtant, la campagne, c'est le territoire. C'est aussi le souffle des villes. Mais sans être un poète urbain, comme Beausoleil ou Daoust, il faut croire que je suis une fille de la ville!

V. et I. - Une question, pour terminer, qu'on pourrait peutêtre mieux poser à des critiques qu'à l'auteure concernée: quelle perception avez-vous de votre situation dans le champ littéraire québécois?

F. N. - Le champ littéraire québécois est invisible pour moi. Je suis sauvage et je n'ai pas grand désir d'entrer dans le clos. Jean Royer m'a déjà posé la même question en soufflant la réponse. Il m'a demandé si j'étais consciente de faire partie d'une génération de femmes qui écrivent. J'avais répondu que j'en étais consciente. C'était il y a quelques années. En y repensant, je me dis que je ne ressemble à personne, surtout pas aux auteurs que j'aime, et ça ne me dérange pas. Ce qui compte, pour moi, ce n'est pas de pouvoir prendre un verre avec Élise Turcotte, par exemple, mais d'avoir lu son roman - formidable! - , ou l'essai de Marco Micone, Le Figuier enchanté, ou un bon gros Tremblay, ou Alison Lurie ou Sepulveda. On n'apprécie pas un texte parce que son auteur est du même sexe, du même âge, du même patelin que soi! En lisant, on a l'impression de se rapprocher de quelqu'un, quelqu'une, mais quand on écrit, c'est toujours seule. Bien sûr, j'admire Anne Hébert et Jacques Ferron, mais comme on admire des parents: ils sont précieux et intouchables. Mais quelle est ma place par rapport à eux? Aucune idée. J'ai l'impression de ne même pas être dans le portrait. Quant aux autres, je les lis, et, la moitié du temps, ça me nourrit. C'est beaucoup. Mais je fonctionne alors comme une lectrice, pas comme écrivaine. Je ne passe pas mon temps à ramener leurs textes aux miens. Je les consomme, dans la joie, quand c'est possible. Aucun rapport avec moi, écrivant mes petits papiers à Montréal, P.Q. Suis-je une auteure québécoise? Forcément. Mais je laisse aux autres la tâche - ou le plaisir - de faire des rapprochements. Pour moi, il ne s'agit pas tant de définir ce que je fais que de le faire. 